Case Report

\title{
Management of Scleral Thinning : An Alternate Approach
}

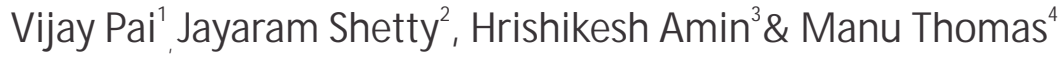 \\ ${ }^{1,3}$ Professors, ${ }^{2}$ Professor \& HOD, ${ }^{4}$ Postgraduate, Department of Ophthalmology, \\ K.S. Hedge M edical Academy, Nitte University, M angalore, Karnataka, India.
}

\section{Correspondence \\ Vijay Pai}

Professor, Department of Ophthalmology, K.S. Hedge M edical Academy, Nitte University, M angalore - 575 108, Karnataka, India.

E-mail : eyevijay@yahoo.com

\begin{abstract}
A 5 year old child presented with corneo-scleral tear in his left eye following trauma with knife which was repaired immediately. 1 month later there was thinning of the sclera at the sutured site. An alternate approach was proposed for the management of scleral thinning. An autologous scleral patch graft from the same eye was sutured at the area of thinning. This method was found to be more convenient and easy to perform. Since the graft was stable and the donor site was healthy; this method can be used as an alternative approach for the management of scleral thinning.
\end{abstract}

Keywords: Scleral thinning, autologous scleral patch graft, Donor sclera

\section{Introduction}

The sclera is the outer fibrous coat which provides a stable support for the intraocular contents. There are various causes for scleral thinning such as, chronic scleritis, scleral injuries following trauma or pterygium surgery ${ }^{1,2}$, excessive use of cautery on scleral bed, over use of antimetabolites like mitomycinfor pterygium and trabeculectomy surgeries $^{3}$, adjunctive irradiation ${ }^{4}$, strabismus surgery, deep sclerotomy procedures, high myopia and systemic vasculitis. ${ }^{1,5}$

In rare instances, it may result in staphyloma formation, scleral perforation, and uveal exposure. Therefore, reinforcement of the thin or perforated sclera is necessary to prevent prolapse of ocular contents and secondary infection.

Various surgical approaches for the management of scleral thinning are donor scleral lamellar patch graft ${ }^{5,6,7}$, multilayered amniotic Access this article online Quick Response Code

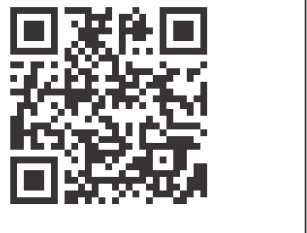
membrane grafting ${ }^{8}$, lamellar corneal graft $^{9}$, rotational scleral graft $^{10}$ and autologous scleral patch graft. $^{11,12}$

An autologous lamellar scleral patch graft from the same eye is described here. The ease of availability of a viable tissue and no risk of rejection were the major advantages. Therefore, this procedure is convenient for emergency cases as well.

\section{Case History}

A 5 year old boy presented to the Department of Ophthalmology with trauma to his left eye with knife. On examination, there was a linear full thickness corneoscleral tear of about $4 \mathrm{~mm}$ in length extending about $3 \mathrm{~mm}$ into the sclera at the 6 'o clock limbus. The corneo-scleral tear repair was done with 10-0 interrupted sutures.

1 month post operatively, a $3 \times 3 \mathrm{~mm}$ area of sclera thinning was noted $2 \mathrm{~mm}$ from the 6 'o clock limbus with ciliary staphyloma. He also developed traumatic cataract. (Figure 1)An autologous scleral patch graft from the patient's own eye with cataract extraction and $\mathrm{PCIOL}$ implantation was planned.

A partial thickness free scleral patch graft of about $3 \times 3 \mathrm{~mm}$ size was fashioned from the upper temporal quadrant at the equatorial region of the sclera of the same eye after raising a fornix based conjunctival flap. The edges of the thinned out sclera at 6 'o clock was freshened after raising the conjunctival flap. The scleral patch graft was carefully positioned at the area of scleral thinning and secured with 10-0 interrupted nylon sutures and was covered with the 
conjunctival flap. In the same sitting, cataract extraction and in the bag PCIOLimplantation was done.

$1^{\text {st }}$ post-operative day, the scleral patch graft was stable. (Figure 2)Patient was discharged on the 3rd day with

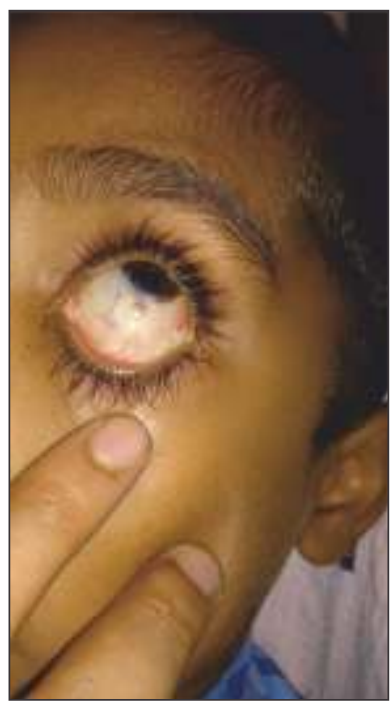

Fig. 1 : Area of thinning $2 \mathrm{~mm}$ below 6 'o clock limbus with ciliary staphyloma (preoperative)

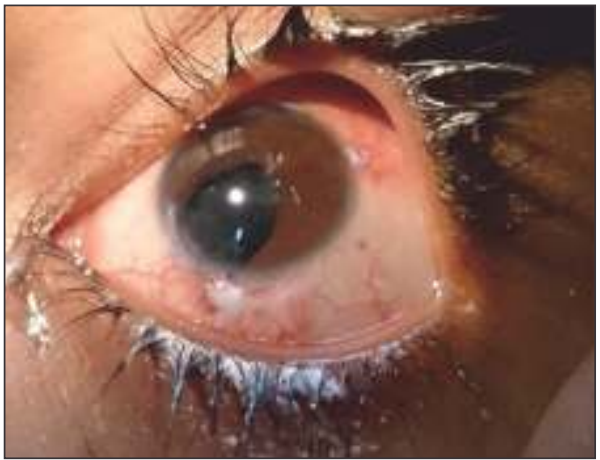

Fig. 2 : postoperative day 1 following scleral patch grafting, cataract extraction and PCIOLimplantation

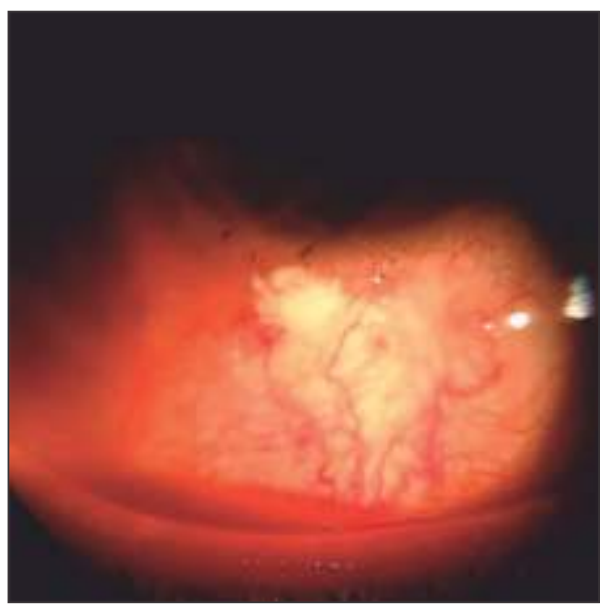

Fig. 3 : A well apposed scleral patch graft 1 month postoperatively

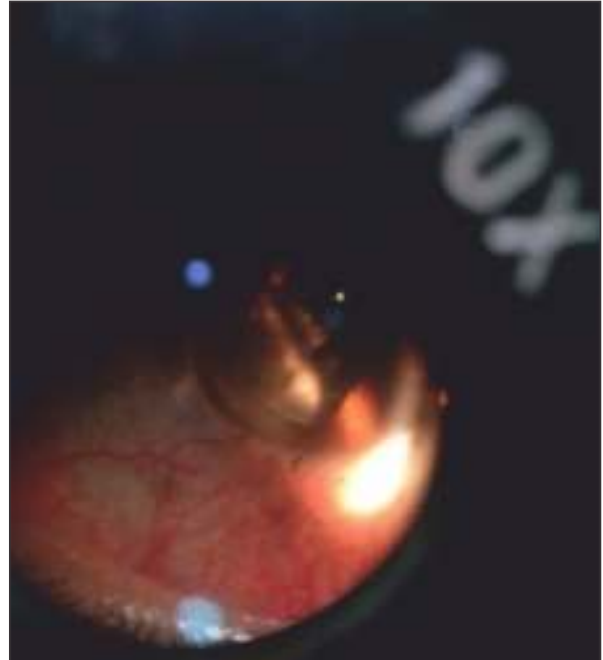

Fig. 4 : Graft taken up well at six weeks postoperative period

systemic antibiotics, analgesics, oral prednisolone $20 \mathrm{mg} /$ day, antibiotic-steroid eye drops hourly and mydriatric eye drops thrice daily. At 1 month follow up, the scleral patch graft was well apposed and there was no signs of uveal tissue exposure.(Figure 3 )

At 6 weeks, the graft uptake was good, the donor site was healthy and there was no evidence of ectasia.(Figure 4) At this point the exposed sutures were removed carefully.

\section{Discussion}

Scleral thinning or melt is a serious and challenging clinical problem as it threatens the integrity of the eye. Different grafts used for scleral thinning include amniotic membrane, sclera, cornea, fascia lata, cartilage, cadaveric aortic tissue, tibial periosteum and skin. ${ }^{1,5.6}$ Literature on autologous scleral graft from same eye is limited. However, various other techniques have been described.

Polat ${ }^{11}$ has described successful repair with autologous lamellar scleral patch graft for scleral melt following pterygium surgery with mitomycin C. Pryda ${ }^{12}$ has reported the repair of peripheral corneal perforation with autologous lamellar scleral patch graft. This procedure is beneficial in emergency cases as there is no hassle of waiting for donor tissue.

A rotational pedicle scleral graft can be done if the area of thinning is small and surrounding sclera is healthy. ${ }^{10}$ In our case, the surrounding sclera was unhealthy, therefore we 
did not opt for a this procedure.

Donor sclera is well-tolerated by the host with little inflammatory reaction and rare rejections and can be preserved for months. ${ }^{1}$ It is strong, flexible and allows a better fit to the host defects. ${ }^{7}$ But complications such as necrosis and melting of graft, dehiscence can be avoided by promoting epithelization and vascularization of the avascular scleral patch graft by covering with conjunctival flap or an amniotic membrane graft. ${ }^{6}$

Ti et $\mathrm{al}^{9}$, reported the use of corneal lamellar graft to maintain integrity of the globe in cases of scleral melting after pterygium surgery. The disadvantage was its transparency making it cosmetically unacceptable to the patient.

Amniotic membrane consists of a thick basement membrane and an avascular stroma. It has antiinflammatory and epithelialization promoting properties. But it may not provide adequate tectonic rigidity and is amenable to rapid disintegration and loss. ${ }^{1} \mathrm{Hwan}$, Kim et al ${ }^{6}$ described scleral grafting with amniotic membrane for

\section{References}

1. V, Jain V, Gupta P. Structural and functional outcome of scleral patch graft. Eye. 2007;21(7):930-5

2. Alsagoff Z, Tan DT, Chee S. Necrotisingscleritis after bare sclera excision of pterygium. British journal of ophthalmology. 2000;84(9):1050-2.

3. Rubinfeld RS, Pfister RR, Stein RM , Foster CS, M artin NF, Stoleru S, et al. Serious complication of topical mitomycin-C after pterygium surgery. Ophthalmology. 1992;99(11):1647-54.

4. Mackenzie FD, Hirst LW, Kynaston B, Bain C. Recurrence rate and complications after beta irradiation for pterygia. Ophthalmology. 1991;98(12):1776-81

5. Nguyen QD, Foster CS. Scleral patch graft in the management of necrotizing scleritis. International ophthalmology clinics. 1999;39(1):109-31.

6. Oh JH, Kim JC. Repair of scleromalacia using preserved scleral graft with amniotic membrane transplantation. Cornea. 2003;22(4):28893.

7. Stunf $S$, Lumi $X$, Drnovšek-Olup B. Preserved scleral patch graft for unexpected extreme scleral thinning found at the scleral buckling procedure: A case report. Indian journal of ophthalmology. 2011;59(3):235. scleromalacia in cases where the adjacent conjunctiva was deficient. Rapid re-epithelialization of ocular surface was noted.

Another rare entity, surgically induced scleral necrosis (SINS), a localised autoimmune reaction occurring at a site of previous surgical wound also caused scleral thinning. It is known to occur after cataract extraction by a limbal incision, strabismus surgery, trabeculectomy and retinal detachment surgery. ${ }^{13,14}$

The limitations of autologous scleral patch graft is the inability to take large grafts to cover large areas of scleral thinning. In cases like high myopes, where the sclera is thin overall, there is a risk of perforation while creating a graft. However, if the sclera is healthy and the area of thinning is small, an autologous lamellar scleral autograft is a safe procedure.

To the best of our knowledge, an autologous lamellar scleral patch graft with cataract extraction and PCIOL implantation in the same sitting is being reported for the first time here.

8. Sridhar M, Bansal A, Rao G. Multilayered amniotic membrane transplantation for partial thickness scleral thinning following pterygium surgery. Eye. 2002;16(5):639-42.

9. Ti S-E, Tan DT. Tectonic corneal lamellar grafting for severe scleral melting after pterygium surgery. Ophthalmology. 2003;110(6):112636

10. Kumar DA, Agarwal A, Nair V, Jacob S, Prakash G, Agarwal A. Rotational Lamellar Scleral Flap for the M anagement of Post trabeculectomy Bleb Leak. Eye \& contact lens. 2013;39(4):e21-e4.

11. Polat N. Use of an Autologous Lamellar Scleral Graft to Repair a Scleral Melt AfterMitomycin Application. Ophthalmology and therapy. 2014:3(1-2):73-6

12. Prydal J. Use of an autologous lamellar scleral graft to repair a corneal perforation. The British journal of ophthalmology. 2006;90(7):924

13. Mahmood S, Suresh P, Carley F, Bruce I, Tullo A. Surgically induced necrotisingscleritis: report of a case presenting 51 years following strabismus surgery. Eye. 2002;16(4):503-4

14. Joseph A, Biswas J, Sitalakshmi G, Gopal L, Badrinath S. Surgically induced necrotisingscleritis (SINS)-Report of two cases. Indian journal of ophthalmology. 1997;45(1):43 